\title{
Backward Bifurcation and the Endemic Equilibrium for an HIV/AIDS - Tuberculosis Co infection Model
}

\author{
Okongo M.O. \\ Physical Sciences department, Chuka University, P.O. Box 60400-109, Kenya.
}

\begin{abstract}
This study proposes a model that describes the dynamics of HIV/AIDS Co infection with Tuberculosis (TB) using systems of nonlinear ordinary differential equations. A characteristic of nonlinear oscillating systems is the sudden change in behavior which occurs as a parameter passes through a critical value called a bifurcation point. A bifurcation point is a point in parameter space where the number of equilibrium points, or their stability properties, or both, change. The results of the study shows that the Co infection model has a diseases-free equilibrium (DFE) which is globally asymptotically unstable implying that the stable endemic state co-exists with the DFE. Numerical simulations are carried out to illustrate the backward bifurcation phenomenon.
\end{abstract}

Keywords: Backward bifurcation, Equilibria, Co-infection, Stability .

DOI: $10.7176 / \mathrm{MTM} / 9-4-05$

Publication date: April $30^{\text {th }} 2019$.

\subsection{Introduction}

A characteristic of nonlinear oscillating systems is the sudden change in behavior which occurs as a parameter passes through a critical value called a bifurcation point. An infectious disease does not invade a population of susceptibles when the basic reproduction number is less than unity. The epidemiological implication of backward bifurcation is that reducing the basic reproduction number to less than unity is not sufficient to control an epidemic (Lawi et al., 2011). When the basic reproduction number is unity, each infectious individual causes one new infection, therefore, whether a disease invades with the basic reproduction number equal to unity will be determined by whether the basic reproduction number increases or decreases as the disease increases along the centre manifold. When backward bifurcation occurs, the DFE may not be globally asymptotically stable even if the basic reproduction number is less than unity and thus a stable endemic state co-exists with the DFE which poses a challenge to the design of effective control measures to be adopted.

\subsection{The Model Equations}

In this study, a deterministic model exploring the joint dynamics of HIV/AIDS and TB co infections at the population level within a single model is developed. The model is described by a system of ordinary differential equations. The total human population $N_{H}(t)$ is the sum of the following epidemiological classes: $S_{H}(t)$ -

Susceptible population at time $\mathrm{t}, \quad I_{H}(t)$ - HIV infected individuals at time t,

$I_{A}(t)$ - AIDS individuals at time $\mathrm{t}, \quad I_{T}(t)$ - TB infected individuals at time $\mathrm{t}, \quad I_{H T}(t)$ - Individuals co infected with HIV and TB at time t, $I_{A T}(t)$ - Individuals co infected with TB and HIV and have progressed to 
AIDS at time t.

$$
\begin{aligned}
& \frac{d S_{H}(t)}{d t}=\Lambda_{H}+r_{t} I_{T}(t)-\lambda_{a h} S_{H}(t)-\lambda_{t h} S_{H}(t)-d_{n} S_{H}(t) \\
& \frac{d I_{H}(t)}{d t}=\lambda_{a h} S_{H}(t)+r_{t} I_{H T}(t)-(1-\alpha) p I_{H}(t)-e_{h}^{t} \lambda_{t h} I_{H}(t) \\
& -d_{n} I_{H}(t)+\alpha I_{A}(t) \\
& \frac{d I_{A}(t)}{d t}=(1-\alpha) p I_{H}(t)+r_{t} I_{A T}(t)-e_{a}^{t} \lambda_{t h} I_{A}(t)-d_{a} I_{A}(t) \\
& -d_{n} I_{A}(t)-\alpha I_{A}(t) \\
& \frac{d I_{T}(t)}{d t}=\lambda_{t h} S_{H}(t)-e_{t}^{h} \lambda_{a h} I_{T}(t)-d_{n} I_{T}(t)-d_{t} I_{T}(t)-r_{t} I_{T}(t) \\
& \frac{d I_{H T}(t)}{d t}=e_{t}^{h} \lambda_{a h} I_{T}(t)+e_{h}^{t} \lambda_{t h} I_{H}(t)-(1-\alpha) \theta_{1} p I_{H T}(t) \\
& -d_{n} I_{H T}(t)-d_{t} I_{H T}(t)-r_{t} I_{H T}(t)+\alpha I_{A T}(t) . \\
& \frac{d I_{A T}(t)}{d t}=e_{a}^{t} \lambda_{t h} I_{A}(t)+(1-\alpha) \theta_{1} p I_{H T}(t)-\alpha I_{A T}(t) \\
& -d_{n} I_{A T}(t)-d_{a} I_{A T}(t)-d_{t} I_{A T}(t)-r_{t} I_{A T}(t)-d_{a t} I_{A T} . \\
& \frac{d N_{H}}{d t}=\Lambda_{H}-d_{n} N_{H}-\left(d_{t} A_{A T}+d_{a} B_{A T}+d_{a t} I_{A T}\right) \\
& A_{A T}=\left(I_{A T}+I_{T}+I_{H T}\right) \text {, and } B_{A T}=\left(I_{A}+I_{A T}\right) .
\end{aligned}
$$

The forces of infection are given by:

$$
\lambda_{a h}=\frac{\beta_{a}(1-\delta) c_{1}\left(I_{H}+I_{H T}\right)}{N_{H}}, \quad \lambda_{t}=\frac{\beta_{t} c_{2}\left(I_{T}+I_{H T}+I_{A T}\right)}{N_{H}}
$$

\subsection{Invariant Region}

The HIV/AIDS-TB model is dissipative: All solutions are uniformly bounded in a proper subset $\left\{\Omega_{H T} \subset i_{+}^{6}\right\}$. Let $\left(S_{H}, I_{H}, I_{A}, I_{T}, I_{A T}, I_{H T}\right) \in i_{+}^{6}$ be any solution with non-negative initial conditions. It

can be easily shown that, all the feasible solutions of system enter the region $\left\{\Omega_{H T} \subset \mathrm{i}_{+}^{6}: N_{H} \leq \frac{\Lambda_{H}}{d_{n}}\right\}$. 
Thus, $\Omega_{H T}$ is positively invariant and attracting and it is sufficient to consider it's solutions in $\Omega_{H T}$. Existence, uniqueness and continuation results for the system hold in this region. Therefore all solutions of the system starting in $\Omega_{H T}$ remain in $\Omega_{H T}$ for all $t \geq 0$. All parameters and state variables for the model are assumed to be non-negative for $t \geq 0$ :

\section{Parameter Values for the HIV/AIDS - TB Co Infection Model}

\begin{tabular}{|c|c|c|c|}
\hline Symbc & Parameter & Value $\left(y r^{-1}\right)$ & Source \\
\hline$\Lambda_{H} \quad \mathrm{R}$ & Recruitment rate of humans & $0.4 \times 40 \times 10^{4}$ & $\begin{array}{l}\text { Kenya demographics } \\
\text { profile (2014) }\end{array}$ \\
\hline$d_{n}$ & Natural death rate of humans & 0.016667 & $\begin{array}{l}\text { Kenya demographics } \\
\text { profile (2014) }\end{array}$ \\
\hline$\alpha$ & $\begin{array}{l}\text { Proportion of the HIV/AIDS } \\
\text { victims treated }\end{array}$ & 0.6 & $\begin{array}{l}\text { Kenya NACC } \\
\text { report }(2014)\end{array}$ \\
\hline$p$ & Progression rate from & 0.1 & Baryama and \\
\hline & HIV to AIDS (untreated) & & Mugisha (2007) \\
\hline$\beta_{a}$ & $\begin{array}{l}\text { Transmission probability } \\
\text { of HIV/AIDS }\end{array}$ & 0.019 & $\begin{array}{l}\text { Baryama and } \\
\text { Mugisha (2007) }\end{array}$ \\
\hline$c_{1}$ & $\begin{array}{l}\text { Per capita number } \\
\text { of sexual contacts }\end{array}$ & 9 & $\begin{array}{l}\text { Kenya NACC } \\
\text { report (2014) }\end{array}$ \\
\hline$\delta$ & Effectiveness of counseling & Variable & \\
\hline$d_{t}$ & Death rate due to TB & 0.02 & Juan and Castillo (2009) \\
\hline$\beta_{t}$ & $\begin{array}{l}\text { Transmission probability of } \\
\text { TB in humans }\end{array}$ & 0.027 & Juan and Castillo (2009) \\
\hline$c_{2}$ & $\begin{array}{l}\text { Contact rate of susceptible } \\
\text { humans with the TB infected }\end{array}$ & 15 & $\begin{array}{l}\text { Juan and } \\
\text { Castillo (2009) }\end{array}$ \\
\hline$\theta_{2}$ & $\begin{array}{l}\text { Increased Progresion rate from } \\
\text { HIV to AIDS due to TB }\end{array}$ & 2 & Estimated \\
\hline$r_{t}$ & $\begin{array}{l}\text { Proportion of TB } \\
\text { victims treated }\end{array}$ & 0.7 & WHO report (2013) \\
\hline$d_{a}$ & HIV/AIDS-induced death rate & 0.4 & WHO report (2014) \\
\hline
\end{tabular}

\subsection{Disease-Free Equilibrium Point of the Model}

The DFE of the model is given by $\varepsilon_{h t}^{0}=S_{\mathrm{H}}^{0} ; I_{\mathrm{H}}^{0} ; I_{\mathrm{A}}^{0} ; I_{\mathrm{T}}^{0} ; I_{\mathrm{HT}}^{0} ; I_{A T}^{0}=\left(\frac{\Lambda_{H}}{d_{n}}, 0,0,0,0\right)$. To study the stability of the DFE, the basic reproduction number $\left(R_{H T}\right)$ which governs the qualitative dynamics of the model is first obtained. Define $F_{i i}$ as the rate of appearance of new infections in the class or compartment $i$ and $v_{i}=v_{i}^{-}-v_{i}^{+}$, where $v_{i}^{-}$is the rate of transfer of individuals out of compartment $\mathrm{i}$, and $v_{i}^{+}$is the rate of 
transfer of individuals into compartment $\mathrm{i}$ by all other means. Therefore the Jacobian of $\mathrm{F}_{i \mathrm{i}}$ and $v_{i}$ at the DFE denoted by $F$ and $V$ respectively is given by:

$$
F=\left(\begin{array}{ccccc}
\beta_{a}(1-\delta) c_{1} & 0 & 0 & \beta_{a}(1-\delta) c_{1} & 0 \\
0 & 0 & 0 & 0 & 0 \\
0 & 0 & \beta_{t} c_{2} & \beta_{t} c_{2} & \beta_{t} c_{2} \\
0 & 0 & 0 & 0 & 0 \\
0 & 0 & 0 & 0 & 0
\end{array}\right)
$$

and

$$
V=\left(\begin{array}{ccccc}
y_{1} & -\alpha & 0 & -r_{t} & 0 \\
-(1-\alpha) p & y_{2} & 0 & 0 & -r_{t} \\
0 & 0 & y_{3} & 0 & 0 \\
0 & 0 & 0 & y_{4} & -\alpha \\
0 & 0 & 0 & -(1-\alpha) \theta_{1} p & y_{5}
\end{array}\right)
$$

Where $y_{1}=(1-\alpha) p+d_{n}, \quad y_{2}=d_{a}+d_{n}+\alpha, \quad y_{3}=d_{a}+d_{t}+r_{t}$ $y_{4}=(1-\alpha) \theta_{1} p+d_{n}+d_{t}+r_{t}, \quad y_{5}=\alpha+d_{n}+d_{a}+d_{t}+d_{a t}+r_{t}$.

The TB reproduction number $R_{T}$ (under treatment) is given by

$$
R_{T}=\frac{\beta_{t} c_{2}}{d_{n}+d_{t}+r_{t}}
$$

The HIV/AIDS reproduction number $\left(R_{H}\right)$ is given by:

$$
\begin{aligned}
& R_{H}=\frac{\beta_{a}(1-\delta) c_{1}\left\{d_{n} p(\alpha-1)+(\alpha-1) \alpha y_{2} p \theta_{1}+\left(y 4\left(y_{5} y_{2}+d_{n} r_{t}\right)\right)\right\}}{\left\{(\alpha-1) \alpha \pi_{1} \theta_{1}-d_{n} p r_{t}+\alpha p r_{t}+y_{4}\left(y_{5} \pi_{1}-d_{n} \pi_{2}\right)\right\}} \\
& \pi_{1}=\alpha d_{n}+d_{a} d_{n}+d_{n}^{2}+d_{a} p-\alpha d_{a} p+d_{n} p-\alpha d_{n} p \\
& \pi_{2}=-d_{n} r_{t}-p r_{t}+\alpha p r_{t} .
\end{aligned}
$$

The reproduction number for the system is given by $R_{H T}=\max \left\{R_{T} ; R_{H}\right\}$. The reproduction number of $T B$ $\left(R_{T}\right)$ shows the number of secondary TB infectious cases produced by a TB infectious individual during his or her infectious period when introduced in a population of susceptibles. The HIV/AIDS reproduction number $\left(R_{H}\right)$ 
in the presence of TB, gives the number of secondary HIV/AIDS infections produced by an HIV/AIDS infectious individual during his or her infectious period when introduced in a population of susceptibles.

Lemma 1. The DFE of the HIV/AIDS-TB model is locally asymptotically stable (LAS) if $R_{H T}<1$, and unstable otherwise.

Proof: Lemma 1 follows from Theorem 2 by Van and Watmough (2002).

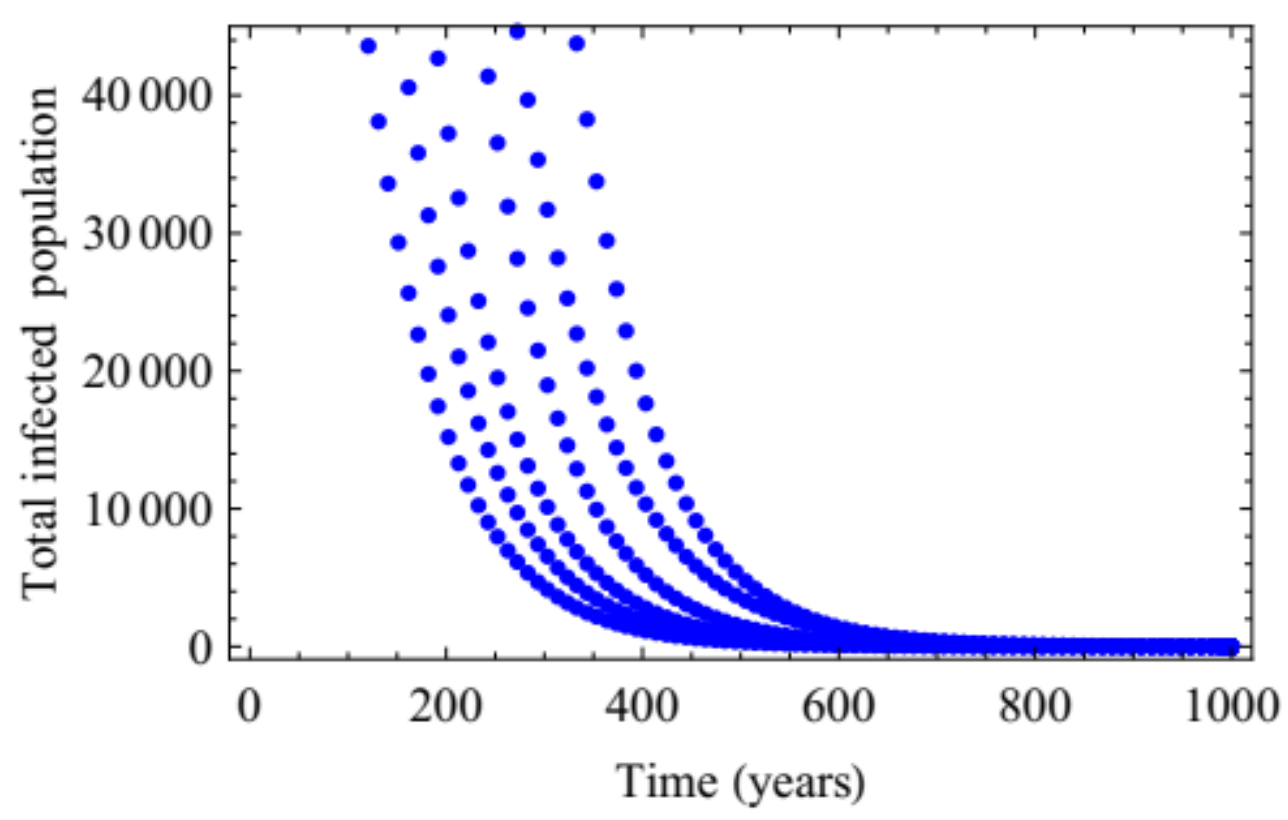

Figure 1: Local Disease Free Equilibrium with $R_{H}=0.60610$ and $R_{T}=0.7015$.

Figure 1 shows the graph of the total infected $\left(\mathrm{I}_{\mathrm{A}}+\mathrm{I}_{\mathrm{H}}+\mathrm{I}_{\mathrm{T}}+\mathrm{I}_{\mathrm{AT}}+\mathrm{I}_{\mathrm{HT}}\right)$ against time in years. The TB reproduction number $R_{T}=0: 6061$ and the HIV/AIDS reproduction number $R_{H}=0.7015$. The local DFE is stable and there is no possibility of further disease outbreaks in future.

\subsection{Global Stability of Disease-Free Equilibrium}

To ensure that elimination of the HIV/AIDS - TB co infections is independent of the initial sizes of the sub-populations, it is necessary to show that the DFE is globally asymptotically stable. The global asymptotic stability (GAS) of the disease-free state of the model is investigated using the theorem by Castillo-Chavez et al. (2002). The model is re written as follows:

$$
\begin{aligned}
& \frac{d X}{d t}=H(X, Z) \\
& \frac{d Z}{d t}=G(X, Z), \quad G(X, 0)=0
\end{aligned}
$$

where the components of the column-vector $X \in \mathrm{i}^{m}$ denote the uninfected population and the components 
of $Z \in \mathrm{i}^{n}$ denote the infected population. Let $E_{0}^{h t}=\left(X^{*}, 0\right)$ denote the DFE of this system. The fixed point $E_{0}^{h t}=\left(X^{*}, 0\right)$ is a globally asymptotically stable equilibrium for this system provided that $R_{0}<1$ and the following two conditions satisfied:

(H1) For $\frac{d X}{d t}=H(X, 0): X^{*}$ is globally asymptotically stable

(H2) $G(X, Z)=P Z-d(X, 0)$, d $(X, 0) \geq 0$, for $(X, Z) \in \Omega_{H T}$,

where $P=D_{Z} G\left(X^{*}, 0\right) \quad$ is an M-matrix (the off diagonal elements of $\mathrm{P}$ are non negative) and $\Omega_{H T} \quad$ is the region where the model makes biological sense. The DFE $E_{0}^{h t}=\left(X^{*}, 0\right)$, where $X^{*}=\left(\frac{\Lambda_{H}}{d_{n}}\right)$

Theorem 1. The fixed point $E_{0}^{h t}=\left(X^{*}, 0\right)$ is a globally asymptotically stable equilibrium of the model provided that $R_{H T}<1$ and the assumptions H1 and H2 are satisfied.

Proof: From the system

$$
\begin{aligned}
& H(X, 0)=\left(\begin{array}{c}
\Lambda_{H}-d_{n} \\
\Lambda_{V}-d_{v}
\end{array}\right) \\
& G(X, Z)=P Z-\widehat{G}(X, Z) \\
& P=\left(\begin{array}{ccccc}
\beta_{1}(1-\delta) c_{1}-y_{1} & \alpha & 0 & r_{t}+\beta_{1}(1-\delta) c_{1} & 0 \\
(1-\alpha) p & -y_{2} & 0 & 0 & r_{t} \\
0 & 0 & \beta_{t} c_{2}-y_{3} & \beta_{t} c_{2} & \beta_{t} c_{2} \\
0 & 0 & 0 & -y_{4} & \alpha \\
0 & 0 & 0 & (1-\alpha) \theta_{1} p & -y_{5}
\end{array}\right)
\end{aligned}
$$

where: $y_{1}=r_{m}+d_{n}+d_{m}, y_{2}=\beta_{a} c_{1}-(1-\alpha) p+d_{n}, y_{3}=d_{a}+d_{n}+\alpha$

$y_{4}=r_{m}+d_{m}+(1-\alpha) p \theta_{1}-d_{n}, y_{5}=r_{m}+d_{m}+\alpha+d_{n}+d_{a}$ 


$$
\widehat{G}=\left(\begin{array}{c}
G_{1}(X, Z) \\
G_{2}(X, Z) \\
G_{3}(X, Z) \\
G_{4}(X, Z) \\
G_{5}(X,)
\end{array}\right)=\left(\begin{array}{c}
\lambda_{a h}\left(1-\frac{S_{H}}{N_{H}}\right)+e_{h}^{t} \lambda_{t h} I_{H} \\
e_{a}^{t} \lambda_{t} I_{A} \\
\lambda_{t h}\left(1-\frac{S_{H}}{N_{H}}\right)+e_{t}^{a} \lambda_{a h} I_{T} \\
-\left(e_{t}^{a} \lambda_{a h} I_{T}+e_{h}^{t} \lambda_{t h} I_{H}\right) \\
-\left(e_{a}^{t} \lambda_{t h} I_{A}\right)
\end{array}\right)
$$

Notice that $\boldsymbol{d}_{4}(X, Z)<0, \boldsymbol{d}_{5}(X, Z)<0$ and so the conditions of $\mathbf{H 1}$ and $\mathbf{H 2}$ are not met so $E_{0}^{h t}$ may not be globally asymptotically stable when $R_{H T}<1$. This implies that there is the possibility of future disease outbreaks when the conditions favouring the outbreaks are prevailing leading to the backward bifurcation phenomenon. This result is numerically illustrated in figure 2 representing the graphs of the total infected population against time in years.

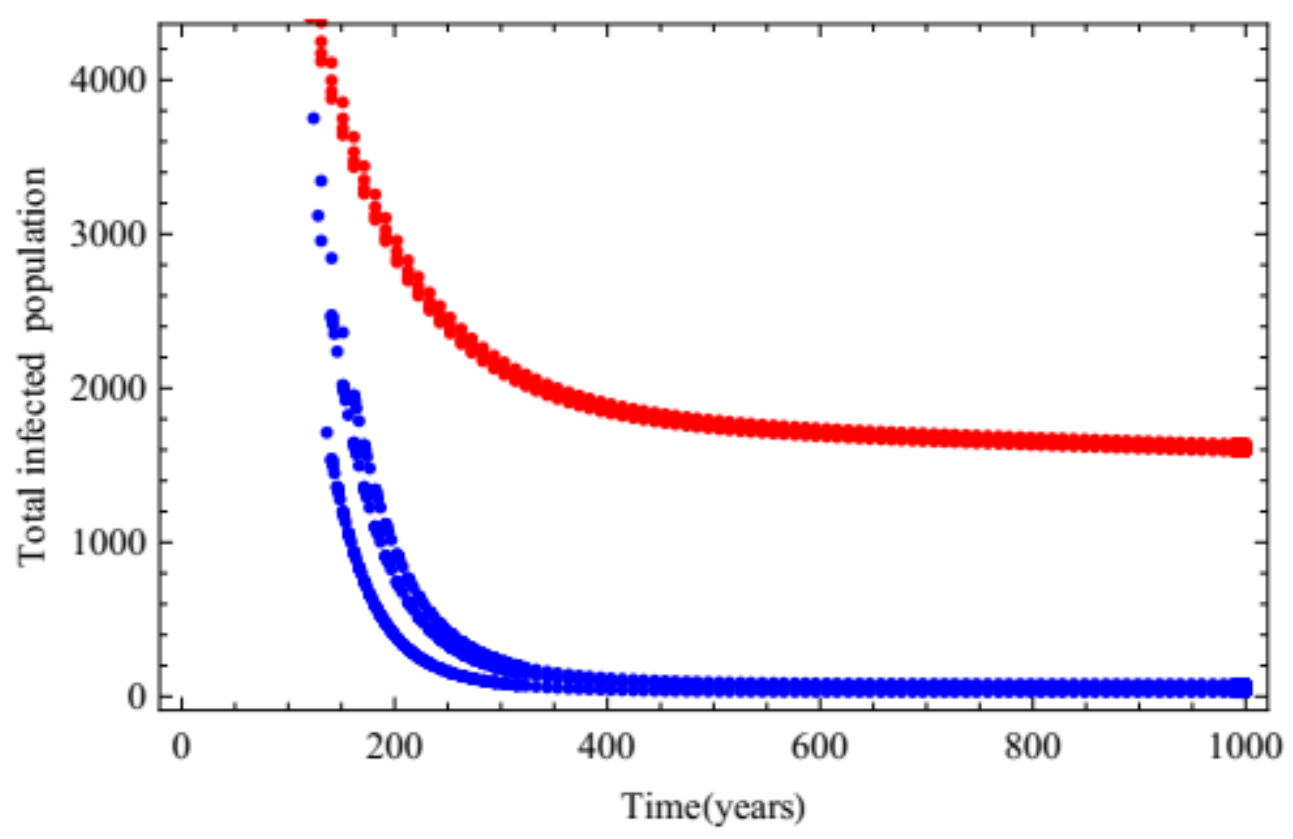

Figure 2: Backward Bifurcation $\left(R_{T}=0.954717\right.$ and $\left.R_{H}=0.997300\right)$.

Figure 2 depicts the phenomenon of backward bifurcation, where multiple stable equilibria co-exists when $R_{H T} \leq 1$. The TB reproduction number $\left(R_{T}\right)=0.954717$ and the HIV/AIDS reproduction number $\left(R_{H}\right)=$ 0.997303 .

\section{Conclusion}

It is noted that the DFE co exist with the endemic equilibrium resulting into the backward bifurcation phenomenon which implies that the classical epidemiological requirement for the eradication of the disease 
whenever $R_{H T}<1$ is no longer sufficient, though necessary which poses a challenge to the design of effective control measures to be adopted.

\section{Acknowledgements}

Thanks to Dr. Lawi George of the department of Mathematics Masinde Muliro university of Science and Technology for his insightful contribution to this work.

\section{REFERENCES}

Baryama, F. and Mugisha, T. (2007). Comparison of single - stage and staged progression models for HIV/AIDS models. International Journal of Mathematics and Mathematical Sciences, 12(4): 399 - 417.

Juan, P. and Castillo, C. (2009). Mathematical modeling of tuberculosis epidemics. Mathematical Biosciences and Engineering, (6) 2: 209 - 237.

Kenya demographics profile. (2014). Accessed on 3rd August 2015 at www.indexm undi.com/kenya/demographicsprofle 2014.

Kenya National AIDS Control Council (NACC) Report.(2014). Accessed on 03/08/ 2015 at http://www.kaisernetwork.org.

Lawi, G. O., Mugisha, J. Y. and Omolo - Ongati, N. (2011), Mathematical model for malaria and meningitis co-infection among children. Applied Mathematical Sciences, Vol. 5: 47, 2337 - 2359

Van, P. and Watmough, J. (2002). Reproduction numbers and the sub-threshold endemic equilibria for compartmental models of disease transmission. Mathematical Biosciences, 180(200): 29-48.

World Health Organization (WHO). (2013). HIV - Associated TB facts: Challenges and key issues. http://www.who.int/tb/challenges/hiv/, Accessed on 13th, August, 2013.

World Health Organization (WHO). (2014a). The World Health Report; HIV/AIDS global maps, Global prevalence of HIV/AIDS, malaria and tuberculosis. ttp://www.google.com/imgres?, Accessed on 5th, February, 2014.

World Health Organization (WHO). (2014b). World Malaria Report 2013; Country profiles. http://www.google.com/imgres?, Accessed on 5th, February, 2014. 